\title{
Primary tumour immune response and lymph node yields in colon cancer
}

\author{
Nikhil Lal $\mathbb{D}^{1 凶}$, Dedrick Kok Hong Chan $\mathbb{D}^{1}{ }^{1}$, Minn E Ng${ }^{2}$, Louis Vermeulen $\mathbb{D}^{3,4}$ and Simon James Alexander Buczacki ${ }^{1,5}$ \\ (c) The Author(s) 2022
}

BACKGROUND: The mechanism underlying improved survival in non-metastatic colon cancer with higher lymph node (LN) yield is unknown. This study aimed to identify whether molecular features in the primary tumour were predictive of LN yield. METHODS: Clinical, genomic, transcriptomic, proteomic and methylation data of non-metastatic, colon cancers studied in The Cancer Genome Atlas were interrogated for associations with LN yield. Based on maximal survival effects, patients were segregated into high (>15) and low ( $\leq 15)$ LN yield. Gene set enrichment analysis was performed on transcriptomic changes to identify biological processes associated with LN yield. Correlations were validated in an independent set of Stage II colon cancers. RESULTS: High LN yield was found predictive of overall and disease-free survival. There was no association of higher LN yield and increasing nodal positivity. High LN yield was strongly linked with gene expression changes associated with the adaptive and dendritic cell immune response. This association was most prominent in node-negative cancers. Analogous findings were reproduced in the validation dataset.

CONCLUSION: The study shows a strong association of an activated immune response in tumours with a high LN yield. Immunogenic tumours have a better prognosis, likely explaining the survival benefit with higher LN yields.

British Journal of Cancer (2022) 126:1178-1185; https://doi.org/10.1038/s41416-022-01700-1

\section{INTRODUCTION}

Lymph node (LN) yield in colon cancer resections is considered crucial for adequate staging and guiding adjuvant treatment [1]. The number of the LNs sampled has also been demonstrated as an independent prognostic marker for improved survival in both node-positive and node-negative disease [2]. Previous studies have highlighted that $\mathrm{LN}$ yield is multifactorial being dependent on surgeon, pathologist and patient-related factors [3, 4]. Currently, the American Joint Commission on Cancer (AJCC) and the National Quality Forum recommend a minimum number of $12 \mathrm{LNs}$ for the adequate staging of colorectal cancers (CRC) [5]. Recently more radical surgical techniques including complete mesocolic excision (CME) with central vessel ligation (CVL) have been developed that improve LN yields in colon cancer resections $[6,7]$.

The mechanism by which higher LN yields are associated with improved survival is unclear. Traditional Halstedian views propose that removal of involved LNs prevents further spread of the disease from these deposits [8]. This view, however, cannot directly explain the clear survival benefit of high LN counts seen in the node-negative disease. In the field of breast cancer surgery, the Cady-Fisher model advocates that LN involvement is a marker of systemic disease and metastasis is influenced by intrinsic tumour-host interactions [9]. The model, therefore, proposes lymphadenectomy for staging alone. Stage migration, also known as the Will Rogers phenomenon, may thus explain the survival benefit seen in node-negative disease with high LN yields [10]. In support, Derwinger et al. observed a trend in stage migration from Stage I/II to Stage III, along with significantly improved survival, in patients with a greater LN yield in CRC resections [11]. However, several studies have contested this theory [12-14]. Bui et al. noted that $\mathrm{LN}$ positivity rates did not increase with a greater $\mathrm{LN}$ yield after colon cancer resections [13]. In 2011, Parsons and colleagues built on this by reporting that node-negative disease and a high LN yield had a lower 5-year mortality compared to node-positive disease [14].

Recently, some groups have hypothesised that a greater LN yield occurs due to an enhanced immune response generated by the host against the tumour $[15,16]$. Markl et al. proposed LN size as a surrogate marker of immune activation, showing that $L N$ size correlated significantly with LN yield and was a prognostic marker in pT3/pT4 node-negative disease [17]. Similarly, other studies have highlighted that lymphocytic and inflammatory cell infiltration in the primary tumour positively impact survival and are associated with the number of nodes harvested [18, 19]. Whilst these studies are useful contributions, it remains unclear whether the weak correlations seen are primary or secondary effects partly

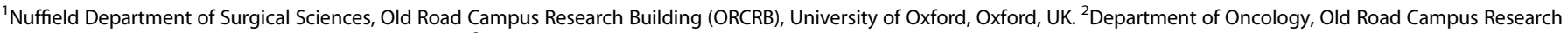

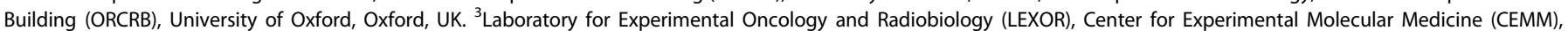

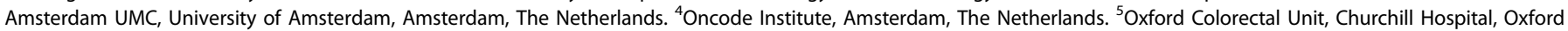
University Hospitals NHS Trust, Old Road, Oxford, UK. ${ }^{凶}$ email: Nikhil.lal@conted.ox.ac.uk

Received: 18 August 2021 Revised: 1 December 2021 Accepted: 6 January 2022

Published online: 18 January 2022 
because of the limited and pre-selected number of measured parameters.

The advent of large, highly annotated multiomic datasets provides a unique opportunity for unbiased bioinformatic analyses to identify tumour molecular correlates with LN yield. Here, we applied this approach to search for biological signatures associated with high $\mathrm{LN}$ yield. Using a discovery and validation dataset, we interrogated genomic, transcriptomic and proteomic data to identify innate primary tumour molecular features that are associated with enhanced $L N$ yield in non-metastatic colon cancer.

\section{METHODS}

\section{Discovery set}

The Cancer Genome Atlas (TCGA) for CRC adenocarcinoma (COAD-READ) dataset was used to extract the discovery set [20]. The study was filtered to include samples of non-metastatic colon cancers of Stage I-III, as defined in the 8th edition of the AJCC [5]. Tumours receiving neoadjuvant treatment were excluded from the study due to the possible impact of neoadjuvant therapy on the LN yield [21]. Tumours without annotation on the number of LN were excluded. Data including clinical attributes, overall \& disease-free survival, genomic alterations, mRNA expression, protein expression and DNA methylation were extracted from the selected cohort. The minimum LN yield relating to the greatest survival benefit were generated for different LN cut-offs with Kaplan-Meier (KM) estimates using the $\mathrm{R}$ software package version 4.0.3 [22]. CMS subtypes were determined using the CMScaller R package [23]. MSI tumours were defined as those having a MSIsensor score of $>3.5$.

\section{Statistical analysis}

Data were analysed in $\mathrm{R}$, cBioPortal and Prism v6 [22, 24-27]. Nonparametric data were summarised tested using the Wilcoxon test for paired groups, Chi-squared test or Fisher's exact test for two independent groups and Kruskal Wallis test for three or more groups. Parametric data were summarised using the Student's $t$ test for two-group analyses. For three or more groups ANOVA was performed. Multiple testing was performed using Benjamini-Hochberg FDR correction.

Gene Set Enrichment Analysis (GSEA) was performed using the GSEA 4.1.0 software package [7]. 'Hallmark' gene sets (50 gene sets) were obtained from the molecular signatures database (MSigDB v4.0) [28]. Each 'hallmark' gene set summarises multiple founder sets relating to a specific biological process or state. To obtain a mechanistic overview of increased LN yield, ranked transcriptomic data were analysed against the 'hallmark' gene sets. Upregulated and downregulated gene sets relating to a high and low LN yield were obtained. Normalised enrichment score (NES) for each gene set was evaluated after adjustment for multiple hypothesis testing. Further subgroup analyses were performed by running the ranked transcriptomic gene lists against the 'hallmark' gene sets for node-positive and node-negative disease. A false discovery rate (FDR) of $<0.05$ was treated as a significant event for all the analyses. Ranked gene lists for transcriptomic data for all samples, node-negative and node-positive disease were tested separately against the estimated immune signatures [29] and xCell type signatures [30].

\section{Validation set}

A highly annotated profile of 90 AJCC Stage II colon cancers undergoing curative surgery between 1997 and 2006 at the Academic Medical Centre (AMC), The Netherlands, were included as the validation cohort (AMCAJCCII-90) [31]. Similar to the discovery set, a ranked list of differentially expressed genes between high (>15) and low ( $\leq 15)$ LN yield samples were generated (GSE33113) using GEO2R and tested against the 'hallmark' gene sets, immune signatures and xCell type signatures for enrichment [32].

\section{RESULTS}

High LN yield in colon cancer surgery is positively associated with survival

(TCGA) Colorectal (COAD) data were filtered to include all nonmetastatic, chemotherapy naive, node-negative and node-positive colon cancer resections $(n=377)$ [20]. The median age at diagnosis was 69 (range: 31-90), and a larger proportion of patients were male ( $n=200,53.05 \%)$ (Supplementary Table 1). There were 230 right-sided tumours compared to 147 left-sided tumours. All patients were diagnosed with colon cancer during a 15-year period, ranging from 1998 to 2013. Moreover, 356 patients had information on the number of LN examined and the median number of LNs retrieved was 20 (range: 2-108) (Fig. 1a). In patients with an adequate $\mathrm{LN}$ assessment $(\geq 12)$ there was no association with higher LN yield and the number of positive nodes identified (Fig. 1b). Similarly, there was no association found between higher LN yield and being nodal positive for all tumours (Fig. 1c). To define a clinically relevant LN cut-off that segregated patients, survival estimates were generated based on LN yield cut-offs between 10 and 20 (Fig. 1d). Compatible with previous reports it was found that segregating patients (node-negative and -positive) to $\leq 15 \mathrm{LN}(n=105)$ and $>15 \mathrm{LN}(n=251)$ provided the most statistically significant difference in both overall and diseasespecific survival (OS; P: 0.0019, 95\% confidence interval $(\mathrm{Cl})$ : 85.95-109.32 months, chi-square value: 9.575, hazard ratio: 0.459 (95\% Cl: 0.277- 0.76), DFS; P: 0.0037, 95\% Cl: 85.14-109.39 months, chi-square value: 8.394 , hazard ratio: 1.445 (95\% Cl: 1.120-1.863)) (Fig. 1e). TCGA data were then mined to look for statistically significant clinical correlations with $\mathrm{LN}$ yield. Patient weight $(P=$ $0.00007)$, height $(P=0.0281)$ and the absence of vascular invasion $(P=0.024)$ were found positively correlated with $\mathrm{LN}$ yield $>15$; however, on multiple testing only patient weight was found significant ( $q=0.001$ ) (Supplementary Table 2). Importantly, there was no association of $L N$ yield with the presence of microsatellite instability (MSI) (Fig. 1f). Further, there was no association of LN yield with any of the consensus molecular subtypes (CMS) of colorectal cancer including hypermutated CMS1 and the unclassified group (Fig. 1g).

Primary tumour immune response is associated with LN yield Next, molecular changes (somatic mutations, copy number alterations, differentially expressed genes, protein levels and methylation changes) were analysed for associations with LN yield. Of the common CRC driver mutations, APC $(P=0.0188)$, TP53 $(P=0.004), \operatorname{KRAS}(P=0.003)$ and FBXW7 $(P=0.024)$ were found more frequently mutated in low $L N$ yield patients but did not reach statistical significance at multiple testing (Fig. 2a). CLDN7, PRKCB, EEF2 and TFRC protein levels were all found at higher levels in high LN yield tumours $(q=0.0283)$. VRK2 methylation levels were also found at higher levels in high LN yield patients ( $q$ $=0.006$ ). To explore transcriptomic changes, GSEA was employed on a ranked gene list of differentially expressed genes between high and low LN yield cancers. Strikingly, a highly significant enrichment for 'hallmark' gene sets associated with immune response in patients with high $L N$ yield was found including INTERFERON_ALPHA_RESPONSE $(q<0.005)$, INTERFERON_GAMMA_RESPONSE $(q<0.005)$, ALLOGRAFT_REJECTION $(q<0.005)$, and INFLAMMATORY_REPONSE $(q<0.005)$ (Fig. 2b-e and Supplementary Table 3). Gene sets associated with a low LN yield were dominated by processes associated with cellular proliferation including E2F_TARGETS $(q<0.005)$, MYC_TARGETS $(q<0.005)$ and the G2M_CHECKPOINT $(q<0.005)$ (Supplementary Fig. 1). These data suggest that LN yield may be driven by an enhanced immune response in the primary tumour making nodes easier to identify ex vivo rather than intrinsic surgical or pathologist-specific factors.

\section{The tumour immune response in high $\mathrm{LN}$ yield tumours is attenuated when there is nodal involvement}

To further explore these observations, tumours were segregated into node-negative and node-positive groups. KM estimates were generated using a $15 \mathrm{LN}$ cut-off for both groups and an overall survival benefit of high LN yield was found in both (Fig. 3a, b). To ascertain whether there were biological differences in high and low LN yield between node-negative and positive groups, 
a

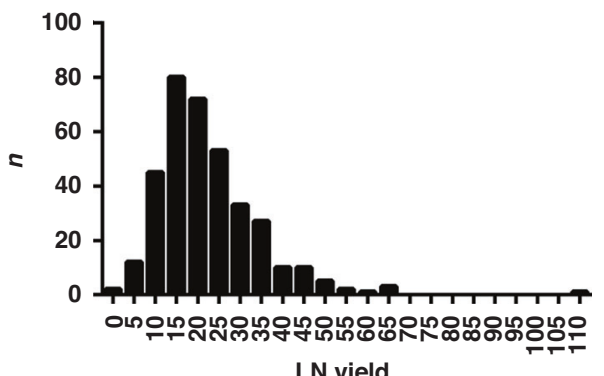

LN yield

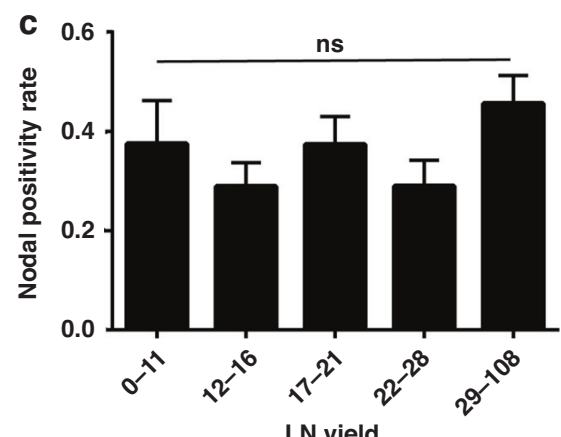

LN yield

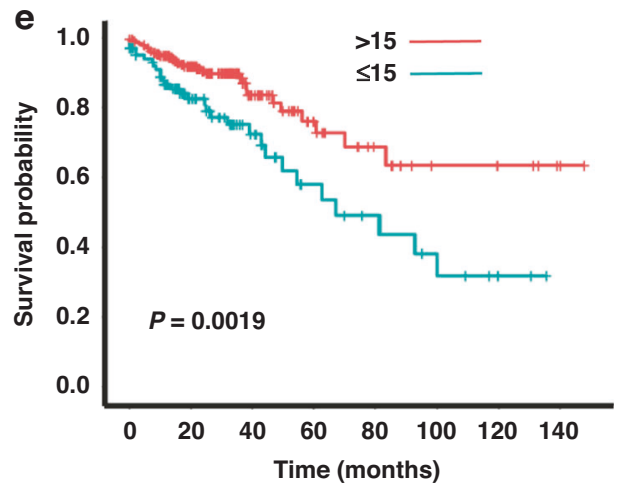

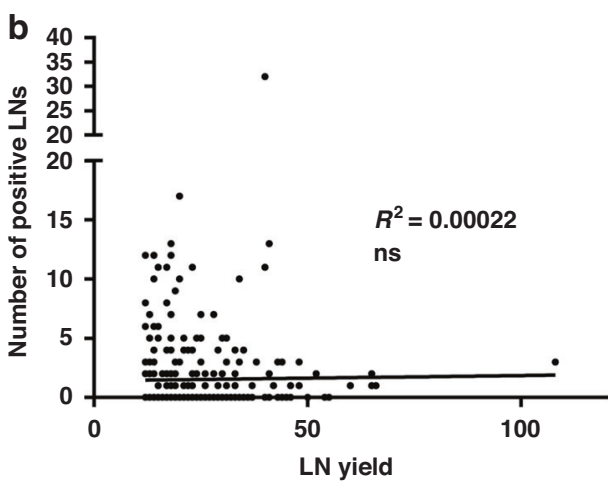

d

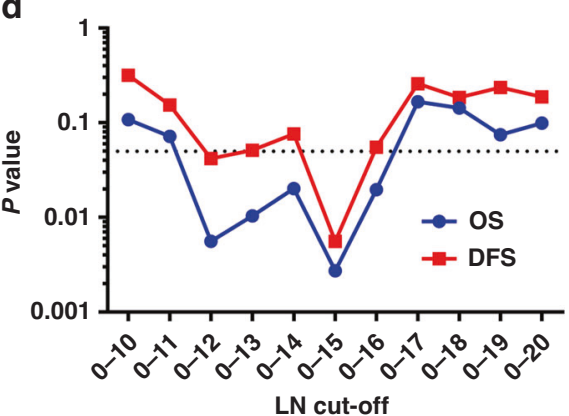

f

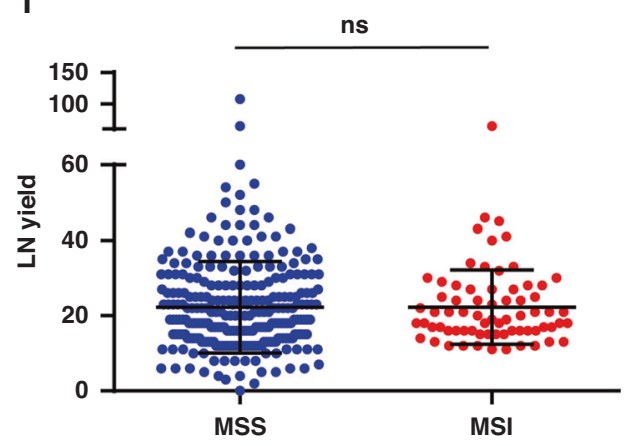

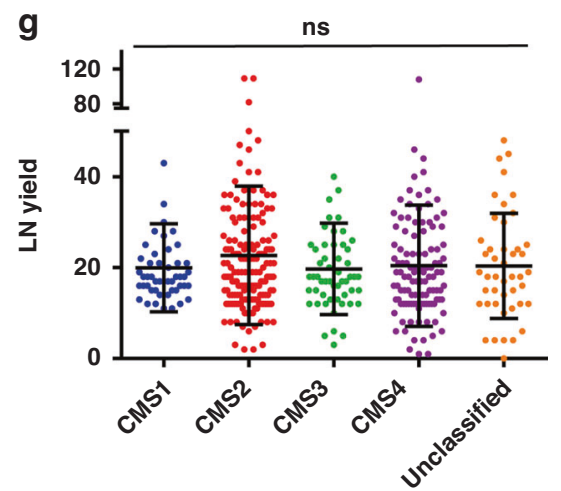

Fig. 1 Stage migration fails to explain the survival benefit of high lymph node yield in TCGA colon cancer resections. a Histogram of LN yield distribution of TCGA-COAD tumours. $\mathbf{b}$ Scatter plot of the association of LN yield with the number of positive LNs found in the specimen. $\mathrm{ns}=$ not significant. $\mathbf{c}$ Histogram of the nodal positivity rate when compared to LN yield. $\mathrm{ns}=$ not significant. $\mathbf{d}$ Significance values for overall and disease-free survival differences for cut-off values of LN yields between 10 and 20. e Kaplan-Meier for the overall survival estimates using a cut-off of 15 LNs. f Column scatter plot comparing lymph node yields between microsatellite stable (MSS) and microsatellite unstable (MSI) colon tumours. $\mathrm{ns}=$ not significant. $\mathbf{g}$ Column scatter plot of LN yields for each consensus molecular subtype (CMS) of colon tumours. $\mathrm{ns}=$ not significant. 


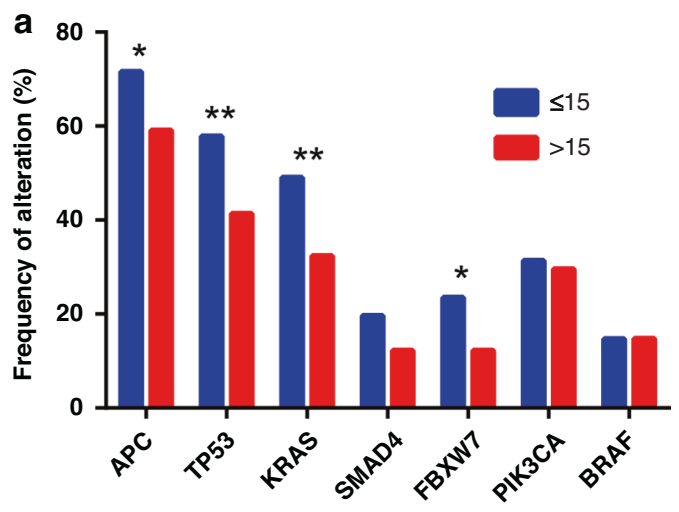

b

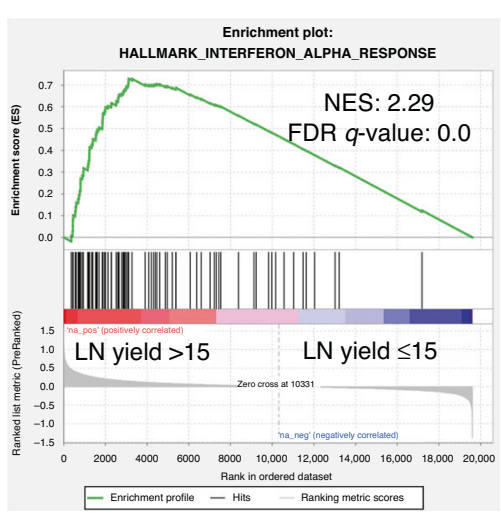

d

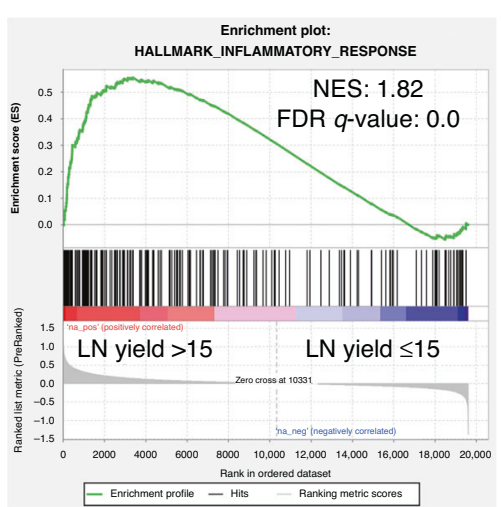

C

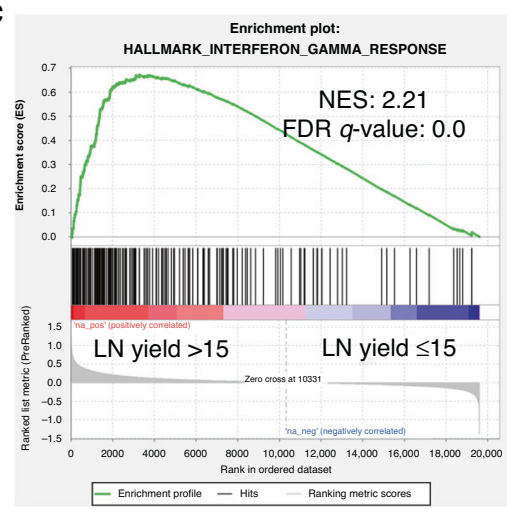

e

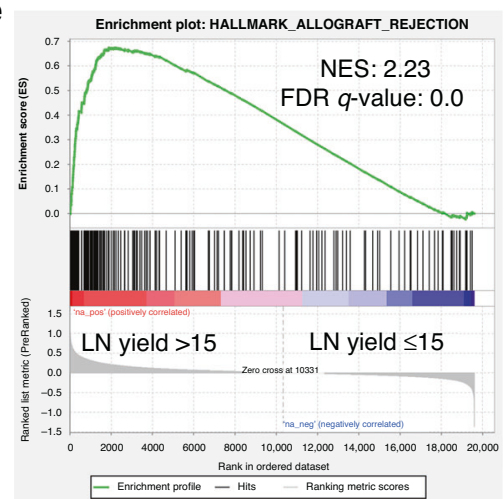

Fig. 2 Hallmark geneset enrichments in high vs low LN yield colon cancers. a Histogram showing the frequency of the most common driver mutations found in tumours with high $(>15)$ and low $(\leq 15)$ LN yields. ${ }^{*} P<0.05,{ }^{* *} P<0.005$. b-e GSEA plots demonstrating the degree of enrichment of the most significant hallmark signatures in high $\mathrm{LN}$ yield tumours.

molecular data from both groups were analysed, using GSEA 'hallmark' gene sets for transcriptomic analysis as previous. There were no genetic alterations, methylation changes or protein expression differences that reached statistical significance for high or low LN yield in both node-negative and node-positive tumours. GSEA however demonstrated very similar findings to the earlier grouped analysis with both LN positive and LN negative tumours showing highly significant associations of immune response with high LN yield (Supplementary Tables 4 and 5). However, it was noted that in all cases the enrichment scores for immune-related gene sets were consistently weaker in node-positive tumours compared to node-negative (Table 1). These findings suggest that the primary tumour immune response that drives high LN yield becomes less effective when tumours have progressed, and LN involvement is present.
High LN yield node-negative tumours have an enhanced adaptive immune response

Theoretically, the association of $L N$ yield with immune response found using GSEA could be driven by two possibilities: high LN yield tumours could have an elevated immune response or low LN yield tumours may have an inadequate response. To explore these options, differentially expressed gene lists were generated for different groups of node-negative tumours. First, the high number of LNs was fixed at $\geq 20$ and the lower cut-off set at $\leq 15, \leq 13$, $\leq 9$ and $\leq 5$. The inflammatory response 'hallmark' gene set showed no change in enrichment scores using GSEA with lower cut-offs suggesting that the immune response in low LN yield tumours does not vary (Fig. 4a). Conversely, when fixing the lower cut-off at $\leq 12$ and changing the higher cut-off to $\geq 25, \geq 30$ and $\geq 35$ a clear trend of increasing enrichment of the inflammation gene set was 

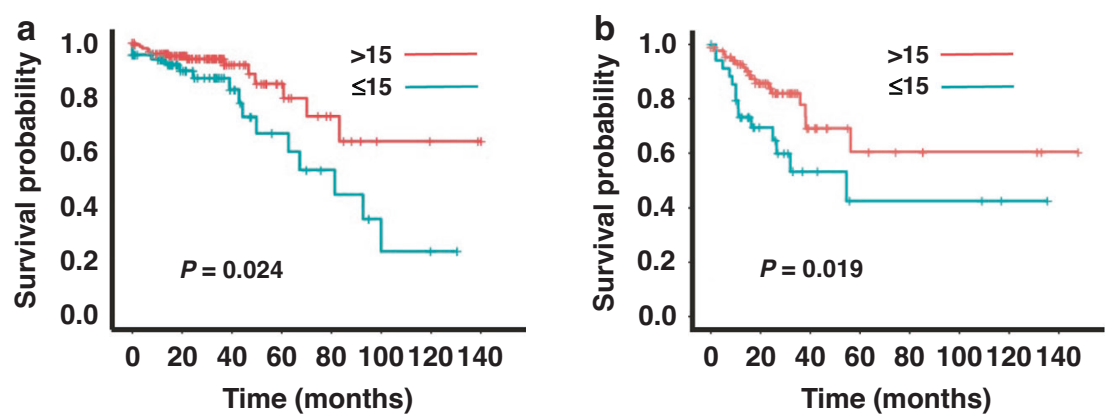

Fig. 3 Kaplan-Meier overall survival (OS) estimates using a cut-off of 15 LNs. a OS for lymph node-negative tumours. b OS for lymph nodepositive tumours. Node-negative; OS; p: 0.024 , 95\% confidence interval (Cl): $81.73-109.18$ months, chi-square value: 5.079 , hazard ratio: 2.25 (95\% Cl: 1.09-4.65). Node-positive; OS; p: 0.019, 95\% Cl: 75.55-111.21 months, chi-square value: 5.478, hazard ratio: 2.276 (95\% Cl: 1.121-4.624).

Table 1. Table of the enrichment scores of immune-related gene sets between node-negative and node-positive high LN yield $(>15)$ tumours.

\begin{tabular}{|lll|}
\hline & Node negative & Node positive \\
\hline INTERFERON_ALPHA & 0.69 & 0.64 \\
\hline INTERFERON_GAMMA & 0.66 & 0.5 \\
\hline INFLAMMATORY_RESPONSE & 0.59 & 0.38 \\
\hline IL2_STAT5 & 0.55 & 0.33 \\
\hline IL6_JAK_STAT3 & 0.6 & 0.29 \\
\hline
\end{tabular}

observed (Fig. 4a). To further explore this all 'hallmark' gene sets were tested against the variable high cut-off tumours. Predictably there was a large overlap of shared gene sets enriched in these high LN groups (30/41). Of these 30 gene sets, 7 showed a significant positive correlation with increasing $L N$ yield: KRAS_SIGNALLING_UP, NOTCH_SIGNALLING, UV_RESPONSE_UP, TGF_BETA_SIGNALLING, INFLAMMATORY_RESPONSE, IL2_STAT5_SIGNALLING and ANDROGEN_RESPONSE (Supplementary Table 6).

The Estimate immune signature and xCell cell-type signatures were next used to independently confirm the apparent high immune response in tumours with high LN yield and explore whether specific subsets of immune cells were enriched in these tumours $[29,30]$. GSEA using the Estimate signature validated the stronger enrichment of immune-related genes in nodenegative high LN yield tumours compared to node-positive (Fig. 4b, c). Analysis of the xCell gene sets showed highly significant enrichment of signatures associated with B cells and T cells (CD4 + and $C D 8+)$ in node-negative tumours with a high LN yield patient suggesting a broad activation of the adaptive immune response in these tumours (Supplementary Table 7). Interestingly, a strong enrichment in dendritic cell signatures was also found. There was a statistically significant enrichment in some gene sets associated with the innate immune response (eosinophils, macrophages, mast cells, basophils, natural killer cells and neutrophils) but this was less strong than the adaptive enrichment.

\section{Validation set}

An independent validation dataset was used to confirm the findings demonstrated using the TCGA data. As Stage II tumours made the largest proportion of our discovery cohort and nodenegative tumours demonstrated greater immunogenicity compared to node-positive, an AMC-AJCCII-90 cohort of a highly annotated transcriptomic dataset of node-negative colon cancers was used as the validation set [31]. Overall, the median age at the time of the operation was 73.4 (range: 34.6-95.1) and there were slightly more female patients than male $(n=42,46.7 \%)$ (Supplementary Table 8 ). A larger proportion of cancers were right-sided $(n=52)$ compared to left-sided $(n=38)$. Of the 90 patients in the group, 84 had associated LN yield numbers. The median number of LNs reviewed were 12 (range: 1-46). Using a cut-off of $15 \mathrm{LNs}$, the cohort was segregated into low yield $(n=56)$ and high yield $(n=$ 28 ) and a ranked differentially expressed gene list generated. GSEA using the 'hallmarks' gene sets demonstrated highly similar findings to that with the TCGA data and strong enrichment of gene sets associated with the immune response (Supplementary Table 9).

\section{DISCUSSION}

Conflicting data around Cady-Fisher or Halstedian explanations lends credence to an alternative explanation for the survival advantage associated with increased $\mathrm{LN}$ yield in colon cancer resections. Here, we approached this important clinical question from an unbiased bioinformatic perspective with the hypothesis that innate biological processes in the primary tumour underlie varying $\mathrm{LN}$ yields. Our approach, using primarily TCGA data, makes use of the largest most highly annotated and well-validated multiomic dataset currently available [20]. Furthermore, the contributions in the TCGA data are multi-institutional, thereby, including centres with varying surgical and pathological techniques to counter-selection bias. Our study reproduces the findings of others showing a clear survival benefit with increased $L N$ yield in both node-negative and node-positive colon cancers [14, 15]. We also find that there is no association between higher LN yields and the number of positive nodes found nor the chance of being node-positive per se. Cumulatively, these findings argue against Halsted, Cady-Fisher and stage-migration mechanisms being an explanation for the profound survival effect seen.

Strikingly, our bioinformatic analysis shows a clear molecular association of enhanced immune response with increasing numbers of LNs retrieved in both node-negative and nodepositive tumours. We hypothesise that a strong immune response in the primary tumour will generate more clearly visible nodes to the pathologist assessing the specimen and thus a higher yield (Fig. 5). Further, this strong immune response against the tumour will result in improved patient survival, as has been shown in many clinical studies [17, 18, 33]. The effect of local immune response on nodal hyperplasia resulting in enlarged lymph nodes has been studied previously [34]. We are not the first to find an association between immune response in the primary tumour and LN yield, however, the two previous studies we cite showed only weak associations and only in T cells $[17,18]$. Kim et al. analysed T-cell makers (CD3, CD8 and CD45RO) finding positive associations with CD3 and CD8 levels and LN yield in Stage II/III colon and rectal resections [18]. Markl et al analysed CD3 and CD8 levels in Stage II colon cancer resections and found a positive association with CD3 levels only [17]. Our unbiased multiomic approach finds a broad B- and T-cell adaptive immune response is the dominant differentially regulated process between high and low LN yield Stage II and III colon cancer resections. We also describe, for the 


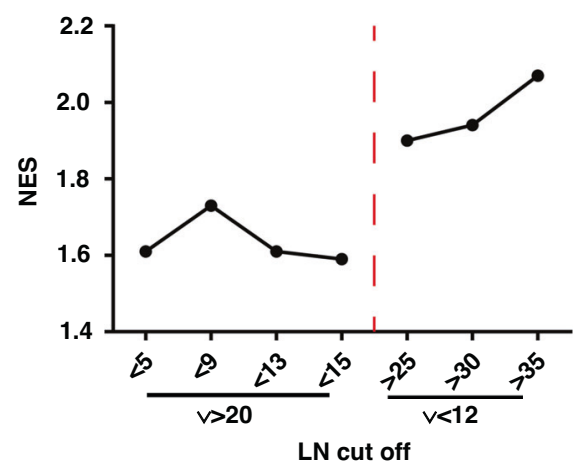

C

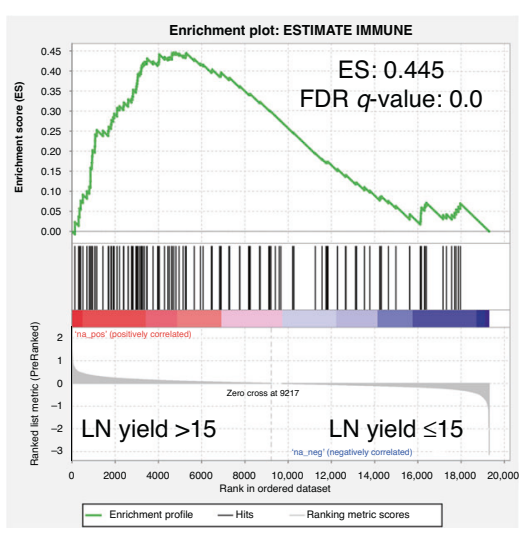

b

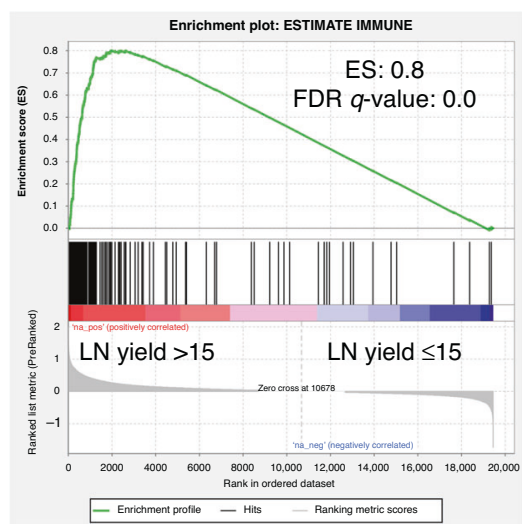

Fig. 4 Relative immune response in node-negative and node-positive colon cancers. a Linked column chart of the inflammation gene set normalised enrichment scores (NES) for different cut-offs of LNs in node-negative colon cancers. b GSEA plot for the Estimate immune signature in node-negative colon cancers ranked by differential expression between high and low LN yield cancers. c GSEA plot for the Estimate immune signature in node-positive colon cancer ranked by differential expression between high and low LN yield cancers.

Low immunogenicity

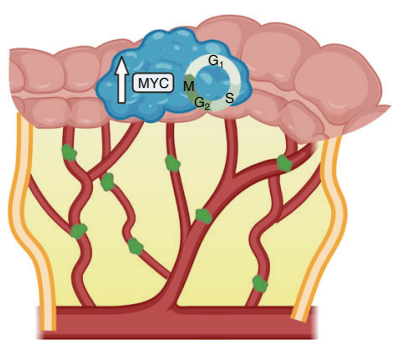

High immunogenicity

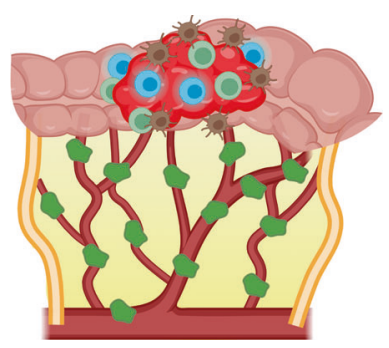

$$
\begin{gathered}
\text { T cell } \\
\begin{array}{c}
\text { Dendritic } \\
\text { cell }
\end{array} \\
\begin{array}{c}
\text { Lymph } \\
\text { node }
\end{array}
\end{gathered}
$$

Fig. 5 Schematic demonstrating the association between tumour immune status and lymph node yield in colorectal cancer.

first time, a strong association between the presence of dendritic cells and high LN yields. This observation is biologically plausible given the well-described role of these cells in transporting cancerassociated antigens to draining LNs for T-cell priming and activation [35]. We find however that this broad immune response becomes attenuated in node-positive cancers. Biologically, this would also be predicted; as a tumour progresses the immune response may act to retard growth and nodal spread. Tumours that subsequently develop nodal involvement would perhaps not unsurprisingly have lost some of that local immune control.

It is interesting that we were unable to identify a clear underlying genetic or epigenetic process driving the apparent enhanced immune response in high LN yield colon cancers. Previous studies have suggested that MSI tumours have an increased LN yield although other groups have not replicated this $[36,37]$. MSI tumours are believed to express high levels of neoantigens driving an enhanced immune response and account for their known susceptibility to checkpoint blockade [38]. In our analysis, we did not find MSI tumours to have higher LN yields, nor any of the recently defined CMS subtypes. These findings are similar to recent studies evaluating the impact of microsatellite instability on LN yield [39]. The contrasting evidence in the literature suggests a need to further study the immunogenicity of MSI tumours. Whilst APC, TP53, KRAS and FBXW7 mutations were more common in low LN yield tumours this was not significant when multiple testing was applied. However, the association of MYC activation and an immunosuppressive environment as seen in low lymph node yield tumours has previously been described in other solid organ malignancies [40]. These findings will provide an important focus for further experimental study in colorectal cancer.

The most exciting recent development in colon cancer surgery has been the development of CME with CVL [6]. Oncological outcome data from the Erlangen group and others are persuasive although have been contested by others [41] and it is uncertain how this radical surgery achieves the apparent benefit [6]. Our study would suggest that the survival effects of these surgeries 
may not be driven by enhanced lymphadenectomy and perhaps rather the exquisite attention to planal surgery and preservation of an intact mesenteric peritoneum, although this hypothesis also remains to be tested. The increasing use of neoadjuvant chemotherapy also has the potential to confound the use of LN yield alone as a prognostic indicator [42]. These therapies have the potential to both perturb the potent antitumour effect of the immune system and may also obscure LN yield estimate postoperatively. Further studies will be required to better understand whether this is a valid concern.

In summary, we show that LN yield in colon cancer resections is determined by a host-tumour adaptive immune response and efforts to maximise LN yield in a contemporary era of pre-existing high-quality surgery and pathology may fail to further improve oncological outcomes.

\section{DATA AVAILABILITY}

Data are available within the article or its Supplementary Materials.

\section{REFERENCES}

1. Ong MLH, Schofield JB. Assessment of lymph node involvement in colorectal cancer. World J Gastrointest Surg. 2016;8:179-92.

2. Le Voyer TE, Sigurdson ER, Hanlon AL, Mayer RJ, Macdonald JS, Catalano PJ, et al. Colon cancer survival is associated with increasing number of lymph nodes analyzed: a secondary survey of intergroup trial INT-0089. J Clin Oncol. 2003;21:2912-9.

3. Tekkis PP, Smith JJ, Heriot AG, Darzi AW, Thompson MR, Stamatakis JD. A national study on lymph node retrieval in resectional surgery for colorectal cancer. Dis Colon Rectum. 2006;49:1673-83.

4. Orsenigo E, Gasparini G, Carlucci M. Clinicopathological factors influencing lymph node yield in colorectal cancer: a retrospective study. Gastroenterol Res. Pr. 2019;2019:5197914.

5. Amin MB, Greene F, Byrd DR, Brookland RK, Washington MK, Gershenwald JE, et al. AJCC cancer staging manual. 8th edn. New York, NY: Springer; 2017.

6. Hohenberger W, Weber K, Matzel K, Papadopoulos T, Merkel S. Standardized surgery for colonic cancer: complete mesocolic excision and central ligationtechnical notes and outcome. Colorectal Dis. 2009;11:354-64.

7. West NP, Hohenberger W, Weber K, Perrakis A, Finan PJ, Quirke P. Complete mesocolic excision with central vascular ligation produces an oncologically superior specimen compared with standard surgery for carcinoma of the colon. J Clin Oncol. 2009;28:272-8.

8. Halsted WSI. The results of operations for the cure of cancer of the breast performed at the Johns Hopkins Hospital from June, 1889, to January, 1894. Ann Surg. 1894;20:497-555.

9. Fisher B. The evolution of paradigms for the management of breast cancer: a personal perspective. Cancer Res. 1992;52:2371-83.

10. Feinstein AR, Sosin DM, Wells CK. The Will Rogers phenomenon. Stage migration and new diagnostic techniques as a source of misleading statistics for survival in cancer. N. Engl J Med. 1985;312:1604-8.

11. Derwinger K, Carlsson G, Gustavsson B. Stage migration in colorectal cancer related to improved lymph node assessment. Eur J Surg Oncol. 2007;33:849-53.

12. Van Erning FN, Crolla RMPH, Rutten HJT, Beerepoot LV, van Krieken JHJM, Lemmens VEPP. No change in lymph node positivity rate despite increased lymph node yield and improved survival in colon cancer. Eur J Cancer. 2014;50:3221-9.

13. Bui L, Rempel E, Reeson D, Simunovic M. Lymph node counts, rates of positive lymph nodes, and patient survival for colon cancer surgery in Ontario, Canada: a population-based study. J Surg Oncol. 2006;93:439-45.

14. Parsons HM, Tuttle TM, Kuntz KM, Begun JW, McGovern PM, Virnig BA. Association between lymph node evaluation for colon cancer and node positivity over the past 20 years. J Am Med Assoc. 2011;306:1089-97.

15. Foo CC, Ku C, Wei R, Yip J, Tsang J, Chan TY, et al. How does lymph node yield affect survival outcomes of stage I and II colon cancer? World J Surg Oncol. 2020;18:22.

16. Sarli L, Bader G, Lusco D, Salvemini C, Mauro DD, Mazzeo A, et al. Number of lymph nodes examined and prognosis of TNM stage II colorectal cancer. Eur J Cancer. 2005;41:272-9.

17. Märkl B, Schaller $T$, Kokot $Y$, Endhardt $K$, Kretsinger $H$, Hirschbühl $K$, et al. Lymph node size as a simple prognostic factor in node negative colon cancer and an alternative thesis to stage migration. Am J Surg. 2016;212:775-80.
18. Kim YW, Jan KM, Jung DH, Cho MY, Kim NK. Histological inflammatory cell infiltration is associated with the number of lymph nodes retrieved in colorectal cancer. Anticancer Res. 2013;33:5143-50.

19. George S, Primrose J, Talbot R, Smith J, Mullee M, Bailey D, et al. Will Rogers revisited: prospective observational study of survival of 3592 patients with colorectal cancer according to number of nodes examined by pathologists. $\mathrm{Br} \mathrm{J}$ Cancer. 2006;95:841-7.

20. cBioPortal. Colorectal Adenocarcinoma (TCGA, Firehouse Legacy). Available from: https://www.cbioportal.org/study/summary?id=coadread_tcga. Accessed 1 May 2021.

21. Amajoyi R, Lee $Y$, Recio PJ, Kondylis PD. Neoadjuvant therapy for rectal cancer decreases the number of lymph nodes harvested in operative specimens. Am J Surg. 2013;205:289-92.

22. The R Project for Statistical Computing. Available from: https://www.r-project.org. Accessed 1 May 2021.

23. Eide PW, Bruun J, Lothe RA, Sveen A. CMScaller: an R package for consensus molecular subtyping of colorectal cancer pre-clinical models. Sci Rep. 2017;7:16618.

24. GraphPad Prism. Available from: https://www.graphpad.com/scientific-software/ prism/. Accessed 1 May 2021.

25. Cerami E, Gao J, Dogrusoz U, Gross BE, Sumer SO, Aksoy BA, et al. The cBio cancer genomics portal: an open platform for exploring multidimensional cancer genomics data. Cancer Discov. 2012;2:401.

26. Gao J, Aksoy BA, Dogrusoz U, Dresdner G, Gross B, Sumer SO, et al. Integrative analysis of complex cancer genomics and clinical profiles using the cBioPortal. Sci Signal. 2013;6:pl1.

27. Subramanian A, Tamayo P, Mootha VK, Mukherjee S, Ebert BL, Gillette MA, et al. Gene set enrichment analysis: a knowledge-based approach for interpreting genome-wide expression profiles. Proc Natl Acad Sci USA. 2005;102:15545.

28. Liberzon A, Birger C, Thorvaldsdóttir H, Ghandi M, Mesirov JP, Tamayo P. The molecular signatures database (MSigDB) hallmark gene set collection. Cell Syst. 2015;1:417-25

29. Yoshihara K, Shahmoradgoli M, Martínez E, Vegesna R, Kim H, Torres-Garcia W, et al. Inferring tumour purity and stromal and immune cell admixture from expression data. Nat Commun. 2013;4:2612.

30. Aran $\mathrm{D}, \mathrm{Hu} Z$, Butte AJ. xCell: digitally portraying the tissue cellular heterogeneity landscape. Genome Biol. 2017;18:220.

31. de Sousa E, Melo F, Colak S, Buikhuisen J, Koster J, Cameron K, et al. Methylation of cancer-stem-cell-associated Wnt target genes predicts poor prognosis in colorectal cancer patients. Cell Stem Cell. 2011;9:476-85.

32. Omnibus GE. AMC colon cancer AJCCII 2011. 2011. Available from: https://www. ncbi.nlm.nih.gov/geo/query/acc.cgi?acc=GSE33113.

33. Ogino S, Nosho K, Irahara N, Shima K, Baba Y, Kirkner GJ, et al. Negative lymph node count is associated with survival of colorectal cancer patients, independent of tumoral molecular alterations and lymphocytic reaction. Am J Gastroenterol. 2010;105:420-33.

34. Son S-M, Woo CG, Lee O-J, Lee S-J, Lee T-G, Lee H-C. Factors affecting retrieval of 12 or more lymph nodes in pT1 colorectal cancers. J Int Med Res. 2019;47:4827-40.

35. Wculek SK, Cueto FJ, Mujal AM, Melero I, Krummel MF, Sancho D. Dendritic cells in cancer immunology and immunotherapy. Nat Rev Immunol. 2020;20:7-24.

36. Berg $M$, Guriby $M$, Nordgård $O$, Nedrebø $B S$, Ahlquist $T C$, Smaaland $R$, et al. Influence of microsatellite instability and KRAS and BRAF mutations on lymph node harvest in stage I-III colon cancers. Mol Med. 2013;19:286-93.

37. Belt EJT, te Velde EA, Krijgsman O, Brosens RPM, Tijssen M, van Essen HF, et al. High lymph node yield is related to microsatellite instability in colon cancer. Ann Surg Oncol. 2012;19:1222-30.

38. Xiao Y, Freeman GJ. The microsatellite instable subset of colorectal cancer is a particularly good candidate for checkpoint blockade immunotherapy. Cancer Discov. 2015;5:16-8.

39. Schrembs $P$, Martin B, Anthuber M, Schenkirsch G, Märkl B. The prognostic significance of lymph node size in node-positive colon cancer. PLoS ONE. 2018;13: e0201072.

40. Jiménez-Sánchez A, Cybulska P, Mager KL, Koplev S, Cast O, Couturier DL, et al. Unraveling tumor-immune heterogeneity in advanced ovarian cancer uncovers immunogenic effect of chemotherapy. Nat Genet. 2020;52:582-93.

41. Olofsson F, Buchwald P, Elmståhl S, Syk I. No benefit of extended mesenteric resection with central vascular ligation in right-sided colon cancer. Colorectal Dis. 2016;18:773-8.

42. Chan DKH, Tan K-K. Lower lymph node yield following neoadjuvant therapy for rectal cancer has no clinical significance. J Gastrointest Oncol. 2018;10:42-7.

\section{ACKNOWLEDGEMENTS}

Dr. Raza Ali (CRUK Cl) for immunological insight and advice on reference datasets. 


\section{AUTHOR CONTRIBUTIONS}

NL: conceptualisation, data curation, formal analysis, investigations, methodology, writing-original draft. DKHC: formal analysis, visualisation, writing-review and editing. MN: writing - original draft. LV: validation, writing - review \& editing. SJAB: conceptualisation, data curation, formal analysis, investigations, methodology, writing - original draft, visualisation and supervision.

\section{FUNDING}

SJAB: The Pharsalia Trust, UK \& Cancer Research UK Advanced Clinician Scientist Fellowship (C14094/A27178). DKHC: Singapore Ministry of Health's National Medical Research Council under a research training fellowship (MOH-FLWSHP10may-0001). LV: New York Stem Cell Foundation-Robertson Investigator. The study sponsors play no roles in the design of the study; the collection, analysis, and interpretation of the data; the writing of the manuscript; or the decision to submit the manuscript for publication.

\section{COMPETING INTERESTS}

The authors declare no competing interests.

ETHICS APPROVAL AND CONSENT TO PARTICIPATE Not applicable.

\section{CONSENT TO PUBLISH}

Not applicable.

\section{ADDITIONAL INFORMATION}

Supplementary information The online version contains supplementary material available at https://doi.org/10.1038/s41416-022-01700-1.

Correspondence and requests for materials should be addressed to Nikhil Lal.

Reprints and permission information is available at http://www.nature.com/ reprints

Publisher's note Springer Nature remains neutral with regard to jurisdictional claims in published maps and institutional affiliations.

Open Access This article is licensed under a Creative Commons Attribution 4.0 International License, which permits use, sharing, adaptation, distribution and reproduction in any medium or format, as long as you give appropriate credit to the original author(s) and the source, provide a link to the Creative Commons license, and indicate if changes were made. The images or other third party material in this article are included in the article's Creative Commons license, unless indicated otherwise in a credit line to the material. If material is not included in the article's Creative Commons license and your intended use is not permitted by statutory regulation or exceeds the permitted use, you will need to obtain permission directly from the copyright holder. To view a copy of this license, visit http://creativecommons. org/licenses/by/4.0/.

(c) The Author(s) 2022 